\title{
THEORETICAL AND EXPERIMENTAL ASPECTS OF SOLAR FLARES MANIFESTATION IN RADIOCARBON ABUNDANCE IN TREE RINGS
}

\author{
A. N. KOSTANTINOV', V. A. LEVCHENKO', G. E. KOCHAROV ${ }^{2}$, I. B. MIKHEEVA ${ }^{2}$ \\ STEFANO CECCHINI ${ }^{3}$, MENOTTI GALLI ${ }^{4}$, TERESA NANNI ${ }^{5}$, PAVEL POVINEC ${ }^{6}$ \\ LIVIO RUGGIERO ${ }^{7}$ and AGOSTINO SALOMONI ${ }^{8}$
}

\begin{abstract}
We describe our method of determining solar cosmic-ray flux and spectrum in the past, based on the comparison of different cosmogenic isotopes. For the period, AD 1781-1950, we have detected several intervals with a high probability of powerful solar flares.
\end{abstract}

\section{INTRODUCTION}

Cosmogenic isotopes can be used as a tool for investigating solar activity in the past. The influence of the Sun on the production of cosmogenic isotopes is two-fold. On the one hand, solar activity modulates the galactic cosmic-ray (GCR) flux, and thus, decreases the cosmogenic isotope production rate. On the other hand, the Sun can generate fluxes of solar cosmic rays (SCR), increasing the cosmogenic isotope production. Until the present, principal interest has been focused on the solar modulation process, as it is the more powerful factor. Nevertheless, the study of solarflare (SF) activity is, without doubt, of special interest for the investigation of, for example, solarterrestrial relations and theories of solar and stellar activity.

\section{BACKGROUND}

Lingenfelter and Hudson (1980) made one of the first attempts to consider the SF activity in the past, based on cosmogenic isotope data. The authors used a strong assumption of constant mean flux of GCR and established the upper limit of the SF activity in the past. The SCR particle spectrum was presented in the form of

$$
\mathrm{dN}_{\mathrm{s}} / \mathrm{dR}=\mathrm{N}_{\mathrm{os}} \exp \left(-\mathrm{R} / \mathrm{R}_{\mathrm{o}}\right)
$$

where $R$ = particle rigidity, $N_{s}=S C R$ flux, $R_{o}=$ characteristic spectrum rigidity, which varies with different flares, and was taken to equal $100 \mathrm{MV}$.

In our opinion, the most effective way of studying solar activity in the past is to use differences of SCR and GCR spectra. Actually, solar particles have much less mean energy than the galactic particles. However, the SCR flux is much higher than the GCR flux. Thus, one has to compare cosmogenic isotope data with different sensitivities for different parts of the particle spectrum. Fortunately, the well-known isotopes, ${ }^{10} \mathrm{Be}$ and ${ }^{14} \mathrm{C}$, are suitable for this purpose. These radionuclides are produced in reactions

$$
{ }^{14} \mathrm{~N}(\mathrm{n}, \mathrm{p}){ }^{14} \mathrm{C} ;{ }^{14} \mathrm{~N}\left(\mathrm{~h},{ }^{5} \mathrm{X}\right){ }^{10} \mathrm{Be} ;{ }^{16} \mathrm{O}\left(\mathrm{h},{ }^{7} \mathrm{X}\right){ }^{10} \mathrm{Be}
$$

\footnotetext{
${ }^{1}$ St. Petersburg State Technical University, St. Petersburg 195251 Russia

${ }^{2}$ Ioffe Physico-Technical Institute, St. Petersburg 194021 Russia

${ }^{3}$ Istituto TESRE-CNR, 40126 Bologna, Italy

${ }^{4}$ Dipartimento di Fisica, Universitá di Bologna, 40126 Bologna, Italy

${ }^{5}$ Istituto FISBAT-CNR, 40126 Bologna, Italy

${ }^{6}$ Department of Nuclear Physics, Comenius University, Bratislava, Czecho-Slovakia

'Dipartimento di Scienza dei Materiali, Universitá di Lecce, 73100 Lecce, Italy

${ }^{8}$ ENEA-TIB, Universitá degli Studi, 40136 Bologna, Italy
} 
where $\mathrm{h}=$ hadrons. The first reaction has practically no energy threshold, so that the ${ }^{14} \mathrm{C}$ and ${ }^{10} \mathrm{Be}$ production rate must have different sensitivities to the galactic and solar particles. Even the production of ${ }^{10} \mathrm{Be}$, which occurs partly in the troposphere and partly in the stratosphere, has different sensitivities to SCR and GCR. In the troposphere, the production of ${ }^{10} \mathrm{Be}$ is caused by primary particles with higher mean energy than for production in the stratosphere. Thus, it is clear that when strong SCR fluxes arrive on the Earth's atmosphere, the ratios of ${ }^{10} \mathrm{Be}$ and ${ }^{14} \mathrm{C}$ production rates can be drastically different.

Figure 1 shows the relation between ${ }^{14} \mathrm{C}$ and ${ }^{10} \mathrm{Be}$ production rates calculated for GCR flux and SCR fluxes with different $R_{0}$. For the GCR, we used the spectrum

$$
\mathrm{dN}_{\mathrm{g}} / \mathrm{dE}=\mathrm{dN}_{\mathrm{og}}\left(E+m c^{2}\right)^{-2.65} \exp (-\mathrm{k} / \mathrm{R})
$$

where $\mathrm{E}=$ the kinetic energy of the particles, $\mathrm{m}=$ the proton mass, $\mathrm{k}=$ the solar modulation parameter. For the solar particles, we assumed the spectrum representation (Eq. 1). The calculation model was described earlier (Kostantinov, Kocharov \& Levchenko 1987). Figure 1 also shows that a large displacement below the curve for GCR might indicate a contribution from the SCR flux to ${ }^{14} \mathrm{C}$ production.

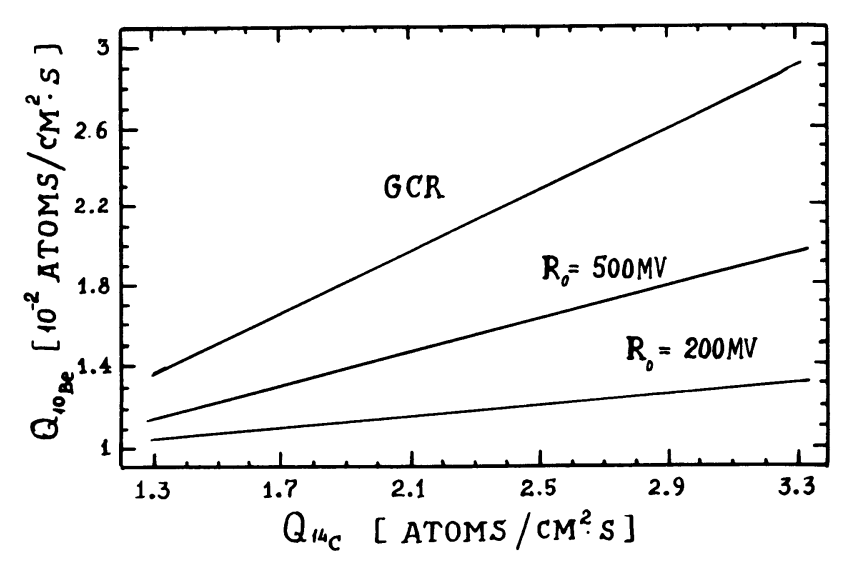

Fig. 1. The relationship between the production rates of ${ }^{14} \mathrm{C}\left({ }^{14} \mathrm{Q}\right)$ and ${ }^{10} \mathrm{Be}\left({ }^{10} \mathrm{Q}\right)$ for GCR and SCR for different characteristic rigidities (MV)

This method has already been tested (Kostantinov, Levchenko \& Mikheeva 1989) on the data of ${ }^{10} \mathrm{Be}$ concentration in an ice core from Greenland, covering the period of the 12th to 17th and 20th centuries $\mathrm{AD}$, and on the corresponding ${ }^{14} \mathrm{C}$ data. Several other periods with possible significant solar flares were also indicated.

In this paper, we present a preliminary attempt to identify solar flares in the past, using recent accurate and detailed data on cosmogenic isotope abundance in terrestrial samples. We used the annual data on ${ }^{10} \mathrm{Be}$ concentration from the Dye 3, Greenland, ice core (Beer et al. 1990), and ${ }^{14} \mathrm{C}$ data (Kocharov et al. 1987b) covering the period, AD 1781-1950. The general procedure for treating the data is described elsewhere (Kostantinov, Levchenko \& Mikheeva 1989). We consider here only the peculiarities of the particular analysis we have carried out.

\section{METHODOLOGY}

Figure 2 shows the temporal profile of ${ }^{10} \mathrm{Be}$ and ${ }^{14} \mathrm{C}$ production rates reconstructed from the original data series. The whole period can be divided into two sections. In the first, from 1781 to 


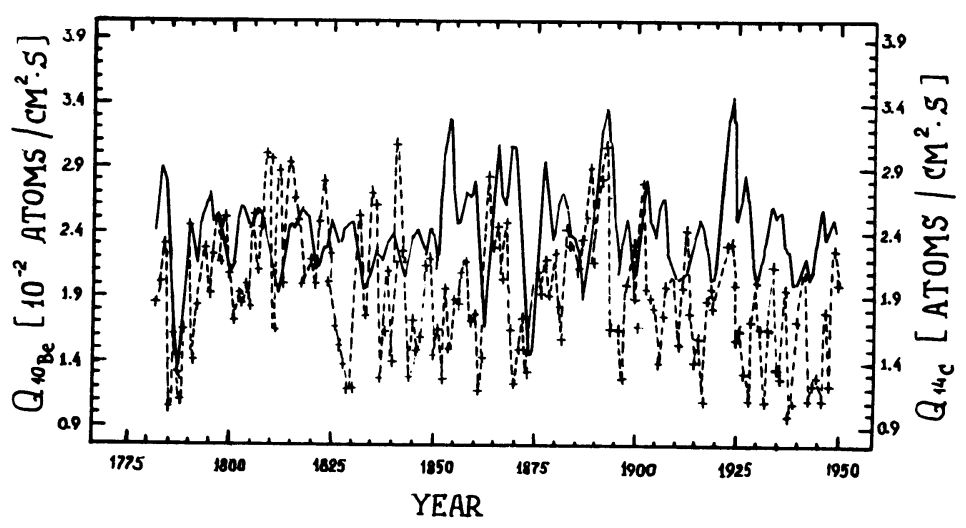

Fig. 2. ${ }^{10} \mathrm{Be}$ and ${ }^{14} \mathrm{C}$ reconstructed production rates: $-={ }^{14} \mathrm{Q}$ in atoms $/ \mathrm{cm}^{2} \mathrm{~s} ; \cdots+\cdots={ }^{10} \mathrm{Q}$ in $10^{-2}$ atoms $/ \mathrm{cm}^{2} \mathrm{~s}$

1900 , the ${ }^{10} \mathrm{Be}$ and ${ }^{14} \mathrm{C}$ profiles are more-or-less similar, and in the second, after $1900,{ }^{10} \mathrm{Be}$ shows a downward trend, with respect to ${ }^{14} \mathrm{C}$. Thus, we decided to consider these two sections separately.

We constructed the cross-correlation function of ${ }^{10} \mathrm{Be}$ and ${ }^{14} \mathrm{C}$ data on the first time interval, and found some discrepancies between the two profiles. Cross-correlation maximum could be achieved by shifting, by one year, one data sequence with respect to the other. We did this to obtain the best fit of the data. As a matter of fact, the ${ }^{14} \mathrm{C}$ exchange system can introduce some uncertainties in the dating of the ${ }^{14} \mathrm{C}$ production rate, and a one-year discrepancy is a good enough fit. It is important to note that the procedure of shifting can reduce the number of discrepancies only among the time profiles, and thus, the number of possible errors in the search for solar flare manifestations.

Figure 3 presents a plot of the ${ }^{10} \mathrm{Be}$ production rate $v s .{ }^{14} \mathrm{C}$ production rate for the period, $\mathrm{AD}$ 1781-1900, together with the theoretical expected dependence and one standard deviation $( \pm 1$ o) strips. The majority of the points is located in this area. According to statistical predictions, one expects about an equal number of points above and below the curve. However, more than twice as many points are below the curve than above. Of course, one can argue that the theoretical expectation is not sufficiently correct, but to that we can reply that our production rate calculations have been tested on experimental data many times before (Kocharov et al. 1987a, 1989). In our opinion, the main cause of discrepancy between the theoretical curve and the point distribution is the presence of an additional factor that was not taken into account in the theoretical calculations, namely, the manifestation of SCR contribution in cosmogenic isotope data. Thus, the average asymmetry of the observed distribution from the theoretical predictions can be interpreted as the influence of the SCR on the production rates, and the average SCR flux can be determined from the value of that divergence. For the period, AD 1781-1900, we obtain the value for SCR flux of $(70 \pm 50)$ particles $/ \mathrm{cm}^{2} \mathrm{~s}$, assuming an average characteristic rigidity of $100 \mathrm{MV}$. The largest uncertainty is due mainly to the ${ }^{14} \mathrm{C}$ reservoir exchange model used for production rate reconstruction. However, our result still does not contradict the modern, generally accepted values.

We can try to explain some divergent points as the manifestation of particular powerful solar flares. Undoubtedly, some of the points have simple statistical origins, such as those that are located in the upper portion. The fact that more than two thirds of the points are in the lower strip seems to indicate that some carry a flare signal. Further, one can see that they are organized in specific temporal groups. We mean that the divergence occurs in groups of years, 2 or 3 sequential years in a group. We have made more precise investigations of these peculiar time intervals by comparing 


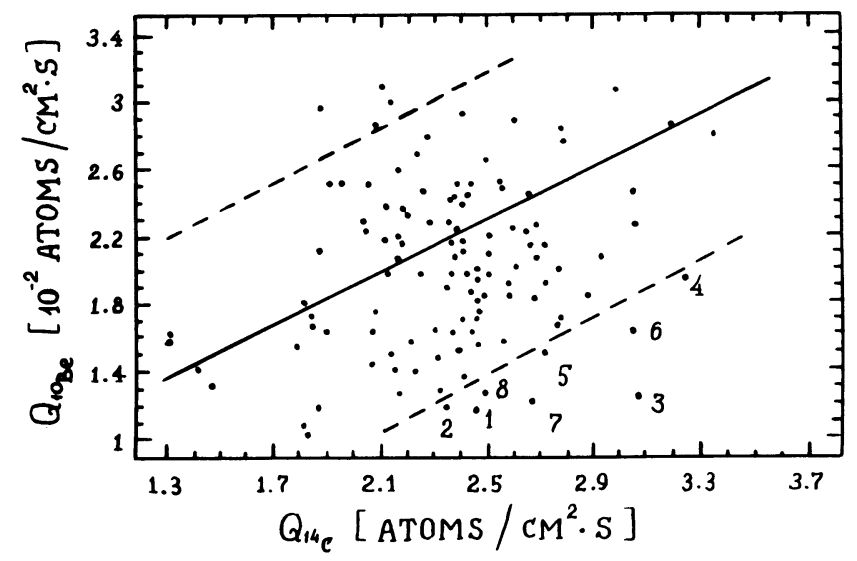

Fig. 3. The observed relationship of reconstructed isotope production rates for the years, AD 1781-1900. $-=$ theoretical correlation for GCR; - - - = regions of \pm 1 o uncertainty. Possible flare years: $1-1824 ; 2-1825 ; 3-1851 ; 4-1852 ; 5-1853$; 6-1869; 7-1870; 8-1897

Wolf sunspot numbers. We found that for three of the mentioned groups, namely at 1851-1853, 1869-1870 and 1897, the divergence can be attributed to strong solar-flare contribution. Figure 4 represents the time behavior of the reconstructed isotopic production rates for the period, AD 1848-1864. One can see that the largest contributions are located on the rising and falling branches of the solar cycles, and that the time profile of production rates shows a certain peculiarity. The same is observed in the other two cases. In our opinion, their explanation as signatures of solarflare contribution is natural.

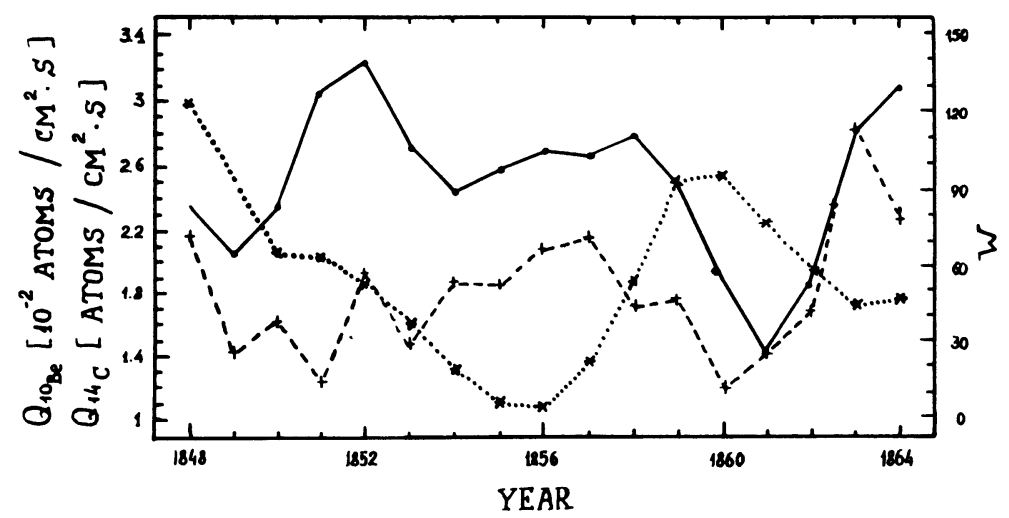

Fig. 4. The comparison of reconstructed isotope production rates (left scale the same as in Fig. 2) and Wolf numbers (right scale) for the years, $1849-1862 .-={ }^{14} \mathrm{Q} ; \ldots={ }^{10} \mathrm{Q} ; \cdots=$ Wolf numbers

For the 1851-1853 event, we estimated the flux and characteristic rigidity. We have assumed an average level of solar modulation, i.e., the value of the coefficient, $k$, in (Eq. 3), because we have only 2 records and 3 unknown values. However, our assumption is not far from the truth, as one can infer by looking at the Wolf numbers. We obtained a solar-flare particle flux of $210^{9}$ particles $/ \mathrm{cm}^{2} \mathrm{~s}$ with $\mathrm{R}_{\mathrm{o}}=200 \mathrm{MV}$. 
The data of the second part, i.e., the data after AD 1900, were analyzed by the same procedure as described above, with one addition. First, we removed the trend from the ${ }^{10} \mathrm{Be}$ data. We believe this is correct because 1) ${ }^{14} \mathrm{C}$ data were also detrended (Suess effect) beforehand, and 2) such detrending can only diminish the number of divergent points, and thus, the number of our errors. The plot of ${ }^{10} \mathrm{Be}$ production rate $v s .{ }^{14} \mathrm{C}$ production rate, together with the sections of uncertainty for the period, AD 1900-1950, is presented in Figure 5. There, also, more points are lying predominantly in the lower portion. As before, we can identify two points, 1923 and 1924, which, with great probability, correspond to enhanced particle fluxes from solar flares. The points corresponding to the mid-1940s lie near the lower boundary of the section, although inside. A strong flare is known to have occurred at that time. Unfortunately, it does not look strong enough to be manifested in our data. Moreover, the year, 1941, is missing from the data sequence.

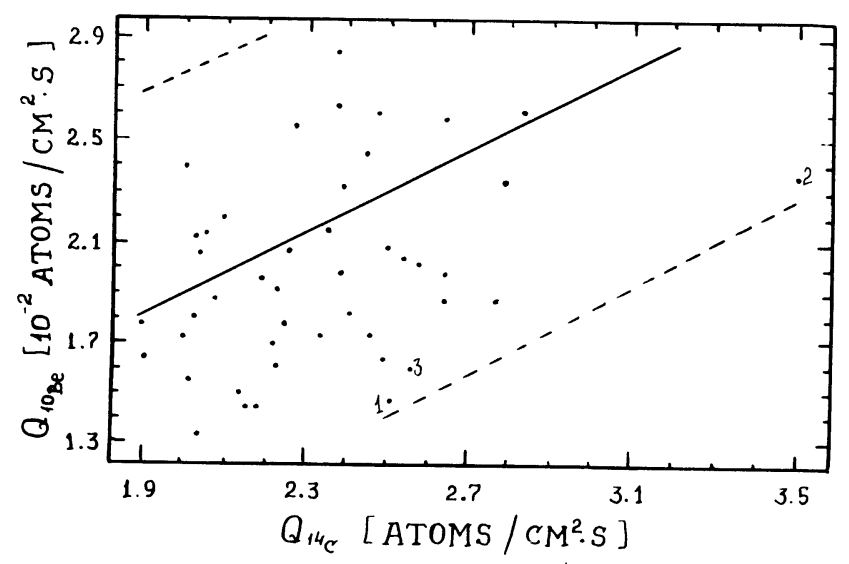

Fig. 5. The comparison of reconstructed isotope production rates for the years, $1900-$ 1950. Legend as in Fig. 3. 1-1925; 2-1924; 3-1946

Shea (1990) made another attempt to find solar-flare particle effects in tree rings ${ }^{14} \mathrm{C}$ abundance for recent flares in September-October 1989.

Because of the non-uniform arrival to the Earth's surface of solar-flare particles due to the anisotropy of the beam, we have looked for local enhancement of ${ }^{14} \mathrm{C}$ production in the northern and southern hemispheres. We measured ${ }^{14} \mathrm{C}$ in samples of wood in which lignin or cellulose supposedly was produced a short time after the flares.

We collected wood samples from a pine tree (Pinus pinea) in Ravenna, Italy $\left(46^{\circ} \mathrm{N}\right.$ Lat) and from a pine tree (Pinus halepensis) in Belair National Park near Adelaide, Australia ( $34^{\circ} \mathrm{S}$ Lat). From both sites, we measured ${ }^{14} \mathrm{C}$ in early wood and late wood, separately, as late wood was supposedly produced even after the stasis of cell differentiation (Attolini et al. 1990), i.e., after September-October in the Ravenna region. A rough correspondence exists between late wood from the northern and early wood from the southern hemispheres.

\section{RESULTS}

Results are shown in Figure 6. Corresponding to the second half of 1989, when a solar-flareparticle effect could be expected, a consistent decrease of $\Delta^{14} \mathrm{C}$ is apparent, instead of an expected increase of $0.5-0.9 \%$, both at northern and southern latitudes, according to the Lingenfelter model. To check the results obtained with scintillation counters of the Bologna Radiocarbon Laboratory, $\Delta^{14} \mathrm{C}$ measurements were also made with proportional counters in Bratislava. The consistency of the measurements is also confirmed in Figure 6. 


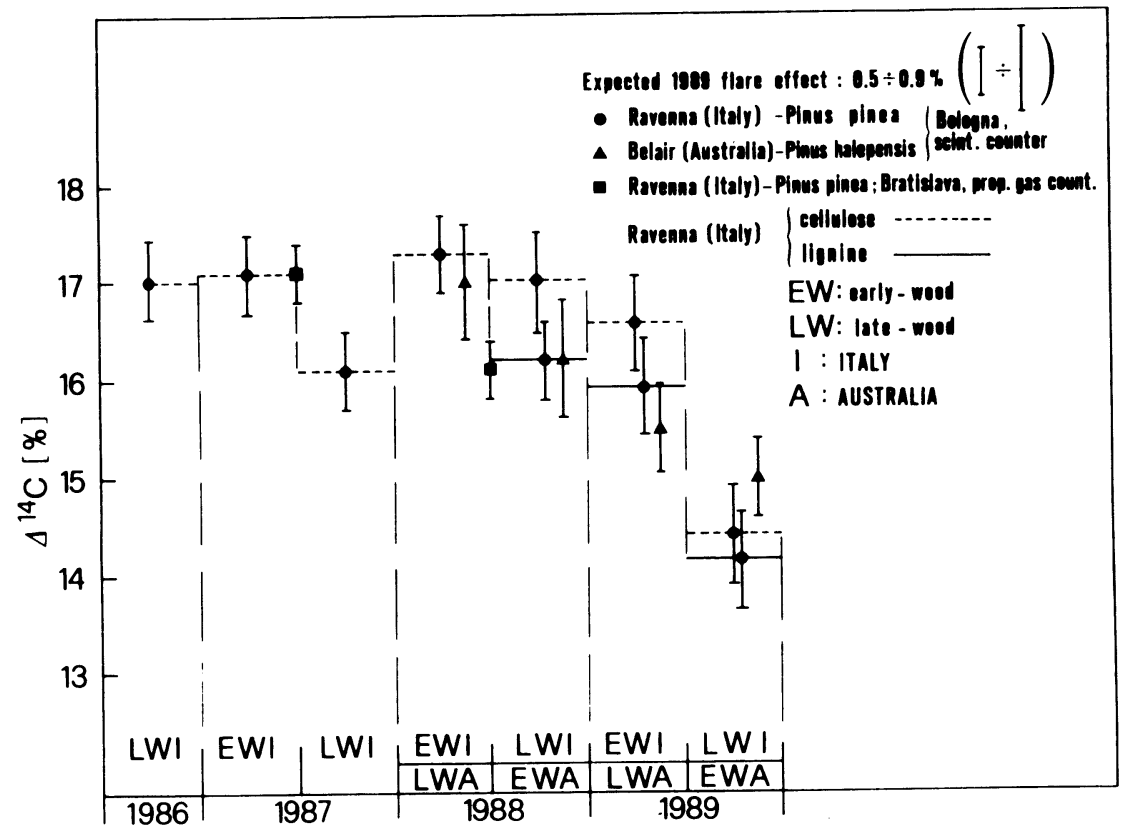

Fig. 6. Results of the search for possible ${ }^{14} \mathrm{C}$ effects in wood caused by solar-flare-particle events of September-October 1989

Thus, we conclude that, as far as the September-October 1989 solar-flare events are concerned, no local anisotropic contribution could be detected.

However, according to our theoretical calculation, a flare effect of the magnitude, $0.5-0.9 \%$, can be expected only in wood of 1990-1991.

\section{CONCLUSIONS}

The main conclusions of this work are:

1. Our suggested method can be used for investigating powerful solar flares in the past, in order to check the frequency distribution of solar-flare events, and to estimate the upper limit of solar energy.

2. In order to obtain more accurate results, we need a third data record, possibly ${ }^{10} \mathrm{Be}$ from high-latitude stations, such as Vostok or Camp Century.

3. Uncertainties can be eliminated by using two isotope data records, e.g. ${ }^{10} \mathrm{Be}$ and ${ }^{26} \mathrm{Al}$, in the same samples from stations at different latitudes.

4. Localized ${ }^{14} \mathrm{C}$ production from the September-October 1989 solar-flare-particle events has not been detected. A diffuse, worldwide ${ }^{14} \mathrm{C}$ effect has not yet been detected. 


\section{REFERENCES}

Attolini, M. R., Calvani, F., Galli, M., Nanni, T., Ruggiero, L., Schaer, E. and Zuanni, F. 1990 The relationship between climatic variable and wood structure in Pinus Halepensis Mill. Theoretical Applications in Climatology 41: 121-127.

Beer, J., Blinov, A., Bonani, G., Finkel, R. C., Hofman, H. J., Lehmann, B., Oeschger, H., Sigg, A., Schwander, J., Staffelbach, T., Stauffer, B., Suter, M. and Wölfli, W. 1990 Use of ${ }^{10} \mathrm{Be}$ in polar ice to trace the 11-year cycle of solar activity. Nature 347: 164-166.

Kocharov, G. E., Blinov, A. V., Kostantinov, A. N. and Levchenko, V. A. 1987a Cosmogenic isotopes: Implications for the cosmic ray spectrum and geomagnetic dipole moment. 20th ICRC, Moscow, Conference Papers 4: 311-314 (in Russian).

1989 Temporal ${ }^{10} \mathrm{Be}$ and ${ }^{14} \mathrm{C}$ variations: $A$ tool for paleomagnetic research. Radiocarbon 31(2): 163168.

Kocharov, G. E., Kostantinov, A. N., Mikheeva, I. B., Bitvinskas, T. T., Metshvarishvili, R. J., Galli, M.,
Cini-Castagnoli, G., Attolini, M. R., Cecchini, S. and Nanni, T. 1987b A 400 year radiocarbon record: 11year and longer cycles. 20th ICRC, Moscow, Conference Papers 4: 280-283 (in Russian).

Kostantinov, A. N., Kocharov, G. E. and Levchenko, V. A. 1987 Cosmogenic isotopes ${ }^{10} \mathrm{Be},{ }^{14} \mathrm{C}$ and ${ }^{36} \mathrm{Cl}$ and astrophysical phenomena. In Kocharov, G. E., ed., Solar Activity and Solar-Terrestrial Relations. Leningrad, LIYAF Publications: 99-134 (in Russian). Kostantinov, A. N., Levchenko, V. A. and Mikheeva, I. B. 1989 On the possibility of the reconstruction of solar cosmic ray parameters in the past. Solnechnye dannye (Solar data) 1: 107-112 (in Russian).

Lingenfelter, R. E. and Hudson, H. S. 1980 Solar particles fluxes and the ancient Sun. In Pepin, R. O., Eddy, J. A. and Merrill, R. B., eds., The Ancient Sun. New York, Pergamon Press: 69-75.

Shea, M. A. 1990 Solar cosmic rays 1960-1989. 21st ICRC, Adelaide 1990, Conference Papers 12: 196201. 